\title{
VARIETIES CORRESPONDING TO CLASSES OF COMPLEMENTED POSETS
}

\author{
IVAN CHAJDA, MIROSLAV KOLAŘÍK, AND HELMUT LÄNGER
}

Received 3 February, 2020

\begin{abstract}
As algebraic semantics of the logic of quantum mechanics there are usually used orthomodular posets, i.e. bounded posets with a complementation which is an antitone involution and where the join of orthogonal elements exists and the orthomodular law is satisfied. When we omit the condition that the complementation is an antitone involution, then we obtain skeworthomodular posets. To each such poset we can assign a bounded $\lambda$-lattice in a non-unique way. Bounded $\lambda$-lattices are lattice-like algebras whose operations are not necessarily associative. We prove that any of the following properties for bounded posets with a unary operation can be characterized by certain identities of an arbitrary assigned $\lambda$-lattice: complementarity, orthogonality, almost skew-orthomodularity and skew-orthomodularity. Moreover, we prove corresponding independence results. Finally, we show that the variety of skew-orthomodular $\lambda$-lattices is congruence permutable as well as congruence regular.
\end{abstract}

2010 Mathematics Subject Classification: 06A11; 06B75; 06C15; 03G12; 08B10

Keywords: bounded poset, complemented poset, orthogonal poset, skew-orthomodular poset, $\lambda$-lattice, variety, congruence distributive, congruence regular

It is well-known that an algebraic semantics of the logic of quantum mechanics is provided by means of orthomodular lattices as shown by G. Birkhoff and J. von Neumann [2] or, independently, by K. Husimi [9]. The details of this construction can be found e.g. in the monograph by L. Beran [1]. However, it was shown later that in the logic of quantum mechanics the connective disjunction represented by the lattice operation $\vee$ need not exist for elements that are not orthogonal. Hence the concept of an orthomodular poset was introduced as follows:

A bounded poset $\mathbf{P}=\left(P, \leq,{ }^{\prime}, 0,1\right)$ with a unary operation is called orthomodular (see e.g. [4]) if ' is an antitone involution on $(P, \leq)$ which is a complementation, i.e. $x \leq y$ implies $y^{\prime} \leq x^{\prime}, x^{\prime \prime}=x, \sup \left(x, x^{\prime}\right)$ exists for all $x \in P$ and it is equal to 1 , and $\inf \left(x, x^{\prime}\right)$ exists for all $x \in P$ and it is equal to 0 ; moreover, $\sup (x, y)$ must exist in case

Support of the research of the first and third author by the Austrian Science Fund (FWF), project I 4579-N, and the Czech Science Foundation (GAČR), project 20-09869L, entitled "The many facets of orthomodularity", as well as by ÖAD, project CZ 02/2019, entitled "Function algebras and ordered structures related to logic and data fusion", and, concerning the first author, by IGA, project PřF 2021 030, is gratefully acknowledged. 
$x \leq y^{\prime}$; finally, for all $x, y \in P$ with $x \leq y$ there exists $x \vee\left(x^{\prime} \wedge y\right)$ and it is equal to $y$. Due to De Morgan's laws, also dually, $y \wedge\left(y^{\prime} \vee x\right)$ exists for all $x, y \in P$ with $x \leq y$ and it is equal to $x$. The last property is called the orthomodular law and can be expressed in the case of lattices alternatively in the form of the equivalent identities

$$
\begin{aligned}
& x \vee\left(x^{\prime} \wedge(x \vee y)\right) \approx x \vee y, \\
& y \wedge\left(y^{\prime} \vee(x \wedge y)\right) \approx x \wedge y .
\end{aligned}
$$

It was shown by V. Snášel [10] that every bounded poset can be organized into a socalled bounded $\lambda$-lattice. Bounded $\lambda$-lattices can be considered as bounded lattices whose binary operations are not necessarily associative. More precisely, a bounded $\lambda$-lattice is a bounded lattice if and only if its binary operations are associative, see [6] for details. The notion of a $\lambda$-lattice was successfully used by the first two authors for constructing a variety of $\lambda$-lattices which corresponds to the class of orthomodular posets. This variety turns out to be congruence permutable and congruence regular. Of course, it is of advantage to work with varieties of algebras instead of classes of posets since for varieties the well-known methods of Universal Algebra can be applied.

Back to the logic of quantum mechanics, we take as an appropriate structure for the algebraic semantics complemented posets in which the join of two orthogonal elements exists and which satisfy the orthomodular law. We do not ask this complementation to be an antitone involution. The concept of complemented lattices which satisfy the orthomodular law, but whose complementation need not be an antitone involution was introduced in [3] and studied in [7]. This concept was generalized by the first and third author to posets in [8]. In the present paper we show that similarly to the case of orthomodular posets, for the posets described above the method of considering assigned $\lambda$-lattices can be successfully applied. In fact, it turns out that important properties of certain posets can be characterized by identities of assigned $\lambda$-lattices.

Let $\mathbf{P}=\left(P, \leq,^{\prime}, 0,1\right)$ be a bounded poset with a unary operation and $a, b \in P$. We define

$$
\begin{aligned}
L(a, b) & :=\{x \in P \mid x \leq a, b\}, \\
U(a, b) & :=\{x \in P \mid a, b \leq x\} .
\end{aligned}
$$

If there exists $\sup (a, b)$ or $\inf (a, b)$ then we will denote these elements by $a \vee b$ or $a \wedge b$, respectively.

We call $\mathbf{P}$ complemented if it satisfies the identities $x \vee x^{\prime} \approx 1$ and $x \wedge x^{\prime} \approx 0$. In this case the operation' is called a complementation. We say that $a, b$ are orthogonal elements of $P$, shortly $a \perp b$, if $a \leq b^{\prime}$. We call a complemented poset $\mathbf{P}$ orthogonal if $x \vee y$ exists for arbitrary orthogonal elements $x, y$ of $P$. We call an orthogonal poset $\mathbf{P}$ almost skew-orthomodular if $x \vee\left(x^{\prime} \wedge y\right)$ exists for all $x, y \in P$ with $x \leq y$. We call 
an almost skew-orthomodular poset $\mathbf{P}$ skew-orthomodular if $x \vee\left(x^{\prime} \wedge y\right)=y$ for all $x, y \in P$ with $x \leq y$.

Example 1. The poset shown in Fig. 1

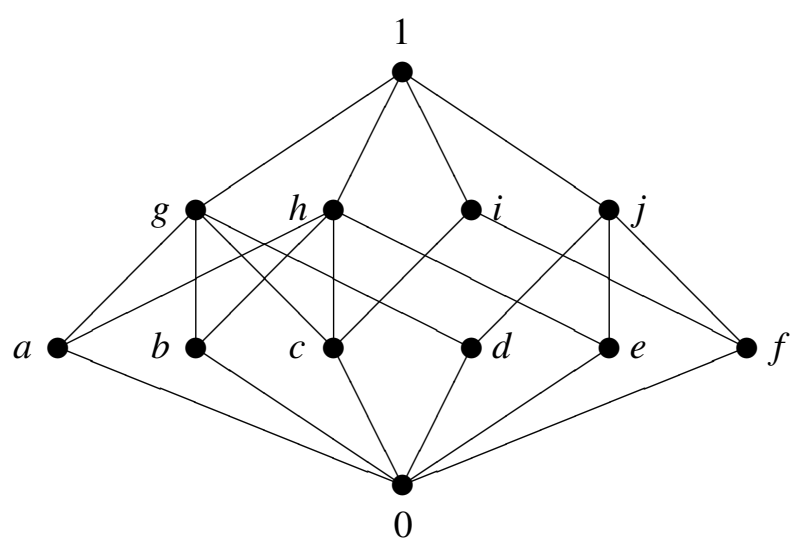

Fig. 1

with

\begin{tabular}{c|cccccccccccc}
$x$ & 0 & $a$ & $b$ & $c$ & $d$ & $e$ & $f$ & $g$ & $h$ & $i$ & $j$ & 1 \\
\hline$x^{\prime}$ & 1 & $j$ & $j$ & $j$ & $i$ & $i$ & $h$ & $f$ & $f$ & $e$ & $c$ & 0
\end{tabular}

is skew-orthomodular, but is not a lattice. Moreover, ${ }^{\prime}$ is antitone, but not an involution.

In the following, let $\mathcal{P}_{c}, \mathcal{P}_{o}, \mathcal{P}_{a}$ and $\mathcal{P}_{s}$ denote the class of all complemented, orthogonal, almost skew-orthomodular and skew-orthomodular posets, respectively. We are going to show that these classes do not coincide, i.e. the inclusions are proper.

Theorem 1. We have

$$
\mathcal{P}_{s} \varsubsetneqq \mathcal{P a}_{a} \mathcal{P}_{o} \varsubsetneqq \mathcal{P}_{c} .
$$


Proof. The poset shown in Fig. 2

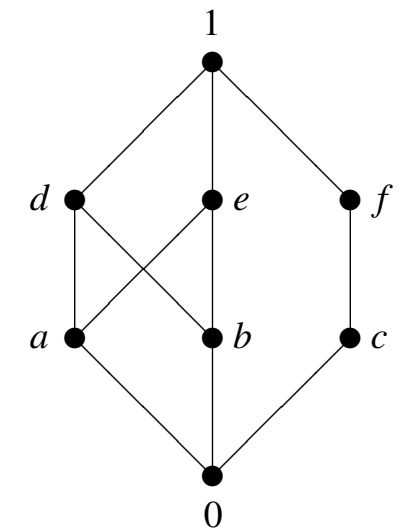

Fig. 2

with

$$
\begin{array}{l|llllllll}
x & 0 & a & b & c & d & e & f & 1 \\
\hline x^{\prime} & 1 & c & c & d & f & f & a & 0
\end{array}
$$

is not a lattice and belongs to $\mathcal{P} a \backslash \mathcal{P}_{s}$ since $a \leq d$, but $a \vee\left(a^{\prime} \wedge d\right)=a \vee(c \wedge d)=$ $a \vee 0=a \neq d$. Moreover, ' is neither antitone nor an involution. The poset shown in Fig. 3

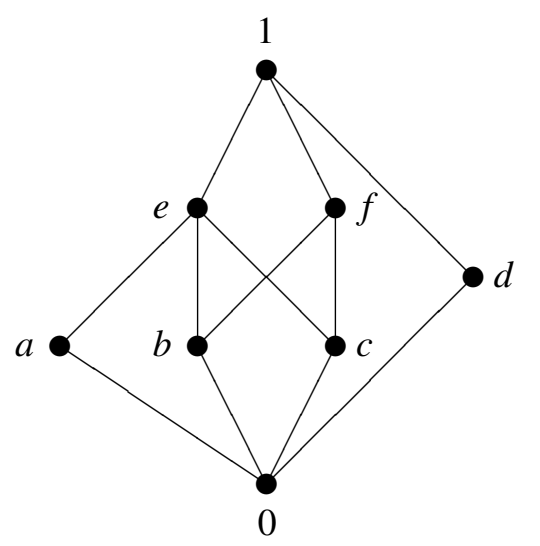

Fig. 3

with

$$
\begin{array}{l|llllllll}
x & 0 & a & b & c & d & e & f & 1 \\
\hline x^{\prime} & 1 & f & d & d & a & d & d & 0
\end{array}
$$


belongs to $\mathcal{P}_{o} \backslash \mathcal{P} a$ since $a \leq e$, but $a^{\prime} \wedge e=f \wedge e$ does not exist. Moreover, ${ }^{\prime}$ is neither antitone nor an involution. The poset shown in Fig. 4

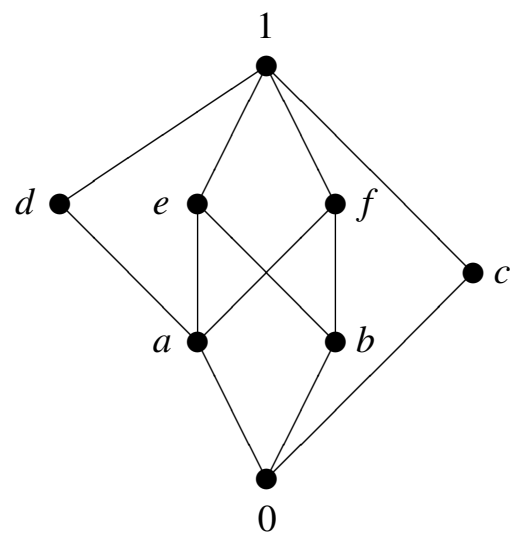

Fig. 4

with

$$
\begin{array}{l|llllllll}
x & 0 & a & b & c & d & e & f & 1 \\
\hline x^{\prime} & 1 & c & d & a & c & c & c & 0
\end{array}
$$

belongs to $\mathcal{P}_{c} \backslash \mathcal{P}_{o}$ since $a \leq d=b^{\prime}$, but $a \vee b$ does not exist. Moreover, ' is neither antitone nor an involution.

Now we introduce the concept of a bounded $\lambda$-lattice taken from [10].

A bounded $\lambda$-lattice is an algebra $(L, \sqcup, \sqcap, 0,1)$ of type $(2,2,0,0)$ satisfying the identities

$$
\begin{aligned}
x \sqcup y & \approx y \sqcup x, x \sqcap y \approx y \sqcap x, \\
x \sqcup((x \sqcup y) \sqcup z) & \approx(x \sqcup y) \sqcup z, x \sqcap((x \sqcap y) \sqcap z) \approx(x \sqcap y) \sqcap z, \\
x \sqcup(x \sqcap y) & \approx x, x \sqcap(x \sqcup y) \approx x, \\
x \sqcup 0 & \approx x, x \sqcup 1 \approx 1 .
\end{aligned}
$$

Hence the class of bounded $\lambda$-lattices forms a variety. Notice that every bounded $\lambda$-lattice satisfies the identities

$$
x \sqcap 0 \approx 0 \text { and } x \sqcap 1 \approx x .
$$

Recall from [6] that every variety of bounded $\lambda$-lattices is congruence distributive. It is well-known that in every bounded $\lambda$-lattice $x \sqcup y=y$ is equivalent to $x \sqcap y=x$.

Let $\mathbf{P}=\left(P, \leq{ }^{\prime}, 0,1\right)$ be a bounded poset with a unary operation. We introduce binary operations $\sqcup$ and $\sqcap$ on $P$ as follows $(x, y \in P$ ): If $x \vee y$ exists then $x \sqcup y:=x \vee y$. Otherwise $x \sqcup y=y \sqcup x$ is an arbitrary element of $U(x, y)$. If $x \wedge y$ exists then $x \sqcap y:=$ $x \wedge y$. Otherwise $x \sqcap y=y \sqcap x$ is an arbitrary element of $L(x, y)$. Then $\left(P, \sqcup, \sqcap,{ }^{\prime}, 0,1\right)$ is a bounded $\lambda$-lattice with a unary operation which we call a $\lambda$-lattice assigned to the bounded poset $\mathbf{P}$. Let $\mathbb{A}(\mathbf{P})$ denote the set of all $\lambda$-lattices assigned to $\mathbf{P}$. 
To every bounded $\lambda$-lattice $\mathbf{L}=\left(L, \sqcup, \sqcap,{ }^{\prime}, 0,1\right)$ with a unary operation we assign a bounded poset $\mathbb{P}(\mathbf{L})=\left(L, \leq{ }^{\prime}, 0,1\right)$ as follows:

$$
x \leq y \text { if and only if } x \sqcup y=y
$$

$(x, y \in L)$. It was shown in [10] that $(L, \leq, 0,1)$ is a bounded poset and

$$
x \leq y \text { if and only if } x \sqcap y=x .
$$

Moreover, using the absorption laws, we easily derive the identities

$$
x \sqcup x \approx x \text { and } x \sqcap x \approx x .
$$

For $i \in\{c, o, a, s\}$ let $\mathcal{L} i$ denote the class of all bounded $\lambda$-lattices $\mathbf{L}$ with a unary operation satisfying $\mathbb{P}(\mathbf{L}) \in \mathcal{P} i$. Hence $\mathcal{L} i$ can be considered as a representation of $\mathcal{P} i$. This means that the properties of $\mathcal{L} i$ may be considered as properties of $\mathcal{P} i$.

In the following we will characterize the above mentioned properties of bounded posets with a unary operation by means of identities of assigned $\lambda$-lattices. Surprisingly, this works despite the fact that this assignment is not unique. Hence, classes of complemented, orthogonal, almost skew-orthomodular and skew-orthomodular posets will be characterized by means of varieties of bounded $\lambda$-lattices. We start with complemented posets.

Theorem 2. Let $\mathbf{P}=\left(P, \leq,{ }^{\prime}, 0,1\right)$ be a bounded poset with a unary operation and $\mathbf{L}=\left(P, \sqcup, \sqcap,{ }^{\prime}, 0,1\right) \in \mathbb{A}(\mathbf{P})$. Then $\mathbf{P}$ is complemented if and only if $\mathbf{L}$ satisfies the identities

$$
\begin{aligned}
& (x \sqcup y) \sqcup\left(x^{\prime} \sqcup y\right) \approx 1, \\
& (x \sqcap y) \sqcap\left(x^{\prime} \sqcap y\right) \approx 0 .
\end{aligned}
$$

Hence $\mathcal{L} c$ is a variety.

Proof. Let $a, b \in P$. First assume $\mathbf{P} \in \mathcal{P} c$. Then

$$
\begin{gathered}
a \leq a \sqcup b \leq(a \sqcup b) \sqcup\left(a^{\prime} \sqcup b\right), \\
a^{\prime} \leq a^{\prime} \sqcup b \leq(a \sqcup b) \sqcup\left(a^{\prime} \sqcup b\right)
\end{gathered}
$$

and hence $(a \sqcup b) \sqcup\left(a^{\prime} \sqcup b\right) \in U\left(a, a^{\prime}\right)=\{1\}$, i.e., $(a \sqcup b) \sqcup\left(a^{\prime} \sqcup b\right)=1$. Dually,

$$
\begin{aligned}
& (a \sqcap b) \sqcap\left(a^{\prime} \sqcap b\right) \leq a \sqcap b \leq a, \\
& (a \sqcap b) \sqcap\left(a^{\prime} \sqcap b\right) \leq a^{\prime} \sqcap b \leq a^{\prime}
\end{aligned}
$$

and hence $(a \sqcap b) \sqcap\left(a^{\prime} \sqcap b\right) \in L\left(a, a^{\prime}\right)=\{0\}$, i.e., $(a \sqcap b) \sqcap\left(a^{\prime} \sqcap b\right)=0$. Conversely, suppose $\mathbf{L}$ to satisfy identities (1) and (2). If $a, a^{\prime} \leq b$ then $a \sqcup b=a^{\prime} \sqcup b=b$ and hence

$$
b=b \sqcup b=(a \sqcup b) \sqcup\left(a^{\prime} \sqcup b\right)=1
$$

showing $a \vee a^{\prime}=1$. Similarly, $b \leq a, a^{\prime}$ implies $a \sqcap b=a^{\prime} \sqcap b=b$ and therefore

$$
b=b \sqcap b=(a \sqcap b) \sqcap\left(a^{\prime} \sqcap b\right)=0
$$


showing $a \wedge a^{\prime}=0$. Hence $\mathbf{P} \in \mathcal{P}_{c}$.

The identities

$$
\begin{aligned}
& x \sqcup x^{\prime} \approx 1, \\
& x \sqcap x^{\prime} \approx 0 .
\end{aligned}
$$

are necessary, but not sufficient for a bounded $\lambda$-lattice to be complemented.

Orthogonal posets can be characterized by an identity that is a bit more complicated than the previous ones (1) and (2).

Theorem 3. Let $\mathbf{P}=\left(P, \leq,{ }^{\prime}, 0,1\right) \in \mathcal{P}_{c}$ and $\mathbf{L}=\left(P, \sqcup, \sqcap,{ }^{\prime}, 0,1\right) \in \mathbb{A}(\mathbf{P})$. Then $\mathbf{P}$ is orthogonal if and only if $\mathbf{L}$ satisfies the identity

$$
\left(\left(\left(x \sqcap y^{\prime}\right) \sqcup z\right) \sqcup(y \sqcup z)\right) \sqcap\left(\left(x \sqcap y^{\prime}\right) \sqcup y\right) \approx\left(x \sqcap y^{\prime}\right) \sqcup y .
$$

Hence Lo is a variety.

Proof. Let $a, b, c \in P$. First assume $\mathbf{P} \in \mathcal{P}_{O}$. Then $\left(a \sqcap b^{\prime}\right) \vee b$ exists. Now

$$
\begin{gathered}
a \sqcap b^{\prime} \leq\left(a \sqcap b^{\prime}\right) \sqcup c \leq\left(\left(a \sqcap b^{\prime}\right) \sqcup c\right) \sqcup(b \sqcup c), \\
b \leq b \sqcup c \leq\left(\left(a \sqcap b^{\prime}\right) \sqcup c\right) \sqcup(b \sqcup c)
\end{gathered}
$$

and hence $\left(\left(a \sqcap b^{\prime}\right) \sqcup c\right) \sqcup(b \sqcup c) \in U\left(a \sqcap b^{\prime}, b\right)$ which yields

$$
\left(a \sqcap b^{\prime}\right) \sqcup b \leq\left(\left(a \sqcap b^{\prime}\right) \sqcup c\right) \sqcup(b \sqcup c)
$$

which is equivalent to identity (3). Conversely, suppose $\mathbf{L}$ to satisfy identity (3). Assume $a \perp b$. Then $a \sqcap b^{\prime}=a$. If $a, b \leq c$ then $a \sqcup c=b \sqcup c=c$ and hence

$$
\begin{aligned}
a \sqcup b & =\left(a \sqcap b^{\prime}\right) \sqcup b=\left(\left(\left(a \sqcap b^{\prime}\right) \sqcup c\right) \sqcup(b \sqcup c)\right) \sqcap\left(\left(a \sqcap b^{\prime}\right) \sqcup b\right) \leq \\
& \leq\left(\left(a \sqcap b^{\prime}\right) \sqcup c\right) \sqcup(b \sqcup c)=(a \sqcup c) \sqcup c=c \sqcup c=c
\end{aligned}
$$

showing $a \sqcup b=a \vee b$, i.e. $a \vee b$ exists. Hence $\mathbf{P} \in \mathcal{P}_{o}$.

Similarly as above we can characterize almost skew-orthomodular posets.

Theorem 4. Let $\mathbf{P}=\left(P, \leq,^{\prime}, 0,1\right) \in \mathcal{P}_{o}$ and $\mathbf{L}=\left(P, \sqcup, \sqcap,{ }^{\prime}, 0,1\right) \in \mathbb{A}(\mathbf{P})$. Then $\mathbf{P}$ is almost skew-orthomodular if and only if $\mathbf{L}$ satisfies the identity

$$
\left(\left((x \sqcap y)^{\prime} \sqcap z\right) \sqcap(y \sqcap z)\right) \sqcup\left((x \sqcap y)^{\prime} \sqcap y\right) \approx(x \sqcap y)^{\prime} \sqcap y .
$$

Hence La is a variety.

Proof. Let $a, b, c \in P$. First assume $\mathbf{P} \in \mathcal{P} a$. Then $(a \sqcap b)^{\prime} \wedge b$ exists. Now

$$
\begin{aligned}
& \left((a \sqcap b)^{\prime} \sqcap c\right) \sqcap(b \sqcap c) \leq(a \sqcap b)^{\prime} \sqcap c \leq(a \sqcap b)^{\prime}, \\
& \left((a \sqcap b)^{\prime} \sqcap c\right) \sqcap(b \sqcap c) \leq b \sqcap c \leq b
\end{aligned}
$$

and hence $\left((a \sqcap b)^{\prime} \sqcap c\right) \sqcap(b \sqcap c) \in L\left((a \sqcap b)^{\prime}, b\right)$ which yields

$$
\left((a \sqcap b)^{\prime} \sqcap c\right) \sqcap(b \sqcap c) \leq(a \sqcap b)^{\prime} \sqcap b
$$


which is equivalent to identity (4). Conversely, suppose $\mathbf{L}$ to satisfy identity (4). Assume $a \leq b$. Then $a \sqcap b=a$. If $c \leq a^{\prime}, b$ then $a^{\prime} \sqcap c=b \sqcap c=c$ and hence

$$
\begin{aligned}
c & =c \sqcap c=\left(a^{\prime} \sqcap c\right) \sqcap c=\left((a \sqcap b)^{\prime} \sqcap c\right) \sqcap(b \sqcap c) \leq \\
& \leq\left(\left((a \sqcap b)^{\prime} \sqcap c\right) \sqcap(b \sqcap c)\right) \sqcup\left((a \sqcap b)^{\prime} \sqcap b\right)=(a \sqcap b)^{\prime} \sqcap b=a^{\prime} \sqcap b
\end{aligned}
$$

showing $a^{\prime} \sqcap b=a^{\prime} \wedge b$, i.e. $a^{\prime} \wedge b$ exists. Moreover, $a \sqcup b=b$. If $a, a^{\prime} \sqcap b \leq c$ then $a \sqcup c=\left(a^{\prime} \sqcap b\right) \sqcup c=c$ and hence

$$
\begin{aligned}
a \sqcup\left(a^{\prime} \sqcap b\right) & =\left(b \sqcap a^{\prime}\right) \sqcup a=\left(\left(\left(b \sqcap a^{\prime}\right) \sqcup c\right) \sqcup(a \sqcup c)\right) \sqcap\left(\left(b \sqcap a^{\prime}\right) \sqcup a\right) \leq \\
& \leq\left(\left(b \sqcap a^{\prime}\right) \sqcup c\right) \sqcup(a \sqcup c)=\left(\left(a^{\prime} \sqcap b\right) \sqcup c\right) \sqcup c=c \sqcup c=c
\end{aligned}
$$

showing $a \sqcup\left(a^{\prime} \sqcap b\right)=a \vee\left(a^{\prime} \wedge b\right)$, i.e. $a \vee\left(a^{\prime} \wedge b\right)$ exists. Hence $\mathbf{P} \in \mathcal{P} a$.

Example 2. The poset shown in Fig. 5

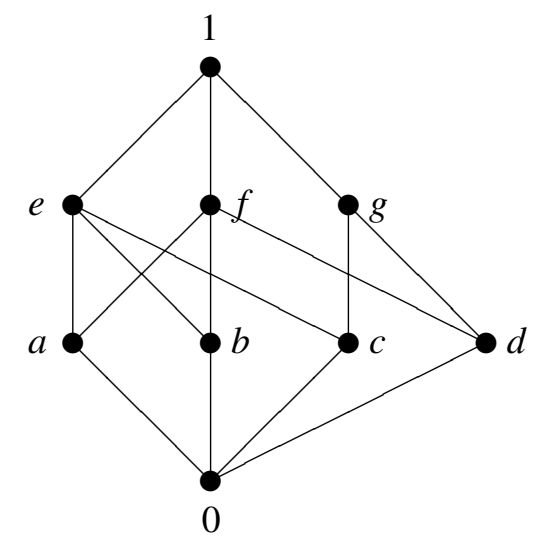

Fig. 5

with

$$
\begin{array}{l|lllllllll}
x & 0 & a & b & c & d & e & f & g & 1 \\
\hline x^{\prime} & 1 & g & g & 1 & 1 & g & g & 1 & g
\end{array}
$$

satisfies identity (4), but does not belong to $\mathscr{P} c$ since $c \wedge c^{\prime}=c \wedge 1=c \neq 0$, and it is not a lattice. Moreover, ' is antitone, but not an involution.

Next we characterize skew-orthomodular posets by identities of assigned $\lambda$-lattices. Since in almost skew-orthomodular $\lambda$-lattices we have

$$
x \sqcup\left(x^{\prime} \sqcap(x \sqcup y)\right)=x \vee\left(x^{\prime} \wedge(x \vee y)\right),
$$

we only need to add a single identity. Let us note that the poset shown in Fig. 1 is almost skew-orthomodular, but not skew-orthomodular. For skew-orthomodularity we have the following result. The proof is evident. 
Corollary 1. Let $\mathbf{P}=\left(P, \leq,^{\prime}, 0,1\right)$ be a bounded poset with a unary operation and $\mathbf{L}=\left(P, \sqcup, \sqcap,{ }^{\prime}, 0,1\right) \in \mathbb{A}(\mathbf{P})$. Then $\mathbf{P}$ is skew-orthomodular if and only if $\mathbf{L}$ satisfies the identities (1) - (5) where

$$
x \sqcup\left(x^{\prime} \sqcap(x \sqcup y)\right) \approx x \sqcup y .
$$

Hence $\mathcal{L} s$ is a variety.

In the following we show some important congruence properties of the variety $\mathcal{L} s$.

Let $\mathcal{V}$ be a variety. The variety $\mathcal{V}$ is called congruence permutable if $\Theta \circ \Phi=$ $\Phi \circ \Theta$ for all $\mathbf{A} \in \mathcal{V}$ and all $\Theta, \Phi \in$ Con $\mathbf{A}$. The variety $\mathcal{V}$ is called congruence regular if for each $\mathbf{A}=(A, F) \in \mathcal{V}, a \in A$ and $\Theta, \Phi \in$ Con $\mathbf{A}$ with $[a] \Theta=[a] \Phi$ we have $\Theta=\Phi$. It is well-known (cf. [5], Theorems 3.1.8 and 6.1.3) that $\mathcal{V}$ is congruence permutable if and only if there exists a so-called Malcev term, i.e. a ternary term $p$ satisfying

$$
p(x, x, y) \approx p(y, x, x) \approx y
$$

and it is regular if and only if there exists a positive integer $n$ and ternary terms $t_{1}, \ldots, t_{n}$ such that

$$
t_{1}(x, y, z)=\cdots=t_{n}(x, y, z)=z \text { if and only if } x=y .
$$

Theorem 5. Let $\mathcal{V}$ be a variety of bounded $\lambda$-lattices $\left(L, \sqcup, \sqcap,{ }^{\prime}, 0,1\right)$ with a unary operation satisfying the identities $x \sqcap x^{\prime} \approx 0$ and (5). Then $\mathcal{V}$ is congruence permutable. In particular, $\mathcal{L} s$ is congruence permutable.

Proof. The term

$$
p(x, y, z):=\left(x \sqcup\left(y^{\prime} \sqcap(y \sqcup z)\right)\right) \sqcap\left(z \sqcup\left(y^{\prime} \sqcap(y \sqcup x)\right)\right)
$$

is a Malcev term since

$$
\begin{aligned}
p(x, x, z) & \approx\left(x \sqcup\left(x^{\prime} \sqcap(x \sqcup z)\right)\right) \sqcap\left(z \sqcup\left(x^{\prime} \sqcap(x \sqcup x)\right)\right) \approx(x \sqcup z) \sqcap\left(z \sqcup\left(x^{\prime} \sqcap x\right)\right) \approx \\
& \approx(x \sqcup z) \sqcap(z \sqcup 0) \approx(x \sqcup z) \sqcap z \approx z, \\
p(x, z, z) & \approx\left(x \sqcup\left(z^{\prime} \sqcap(z \sqcup z)\right)\right) \sqcap\left(z \sqcup\left(z^{\prime} \sqcap(z \sqcup x)\right)\right) \approx\left(x \sqcup\left(z^{\prime} \sqcap z\right)\right) \sqcap(z \sqcup x) \approx \\
& \approx(x \sqcup 0) \sqcap(z \sqcup x) \approx x \sqcap(z \sqcup x) \approx x .
\end{aligned}
$$

We are going to show also congruence regularity of the variety $\mathcal{L} s$.

Theorem 6. Let $\mathcal{V}$ be a variety of bounded $\lambda$-lattices $\left(L, \sqcup, \sqcap,{ }^{\prime}, 0,1\right)$ with a unary operation satisfying the identities $0^{\prime} \approx 1, x \sqcap x^{\prime} \approx 0$ and (5). Then $\mathcal{V}$ is congruence regular. In particular, the variety $\mathcal{L}$ s is congruence regular.

Proof. Put

$$
t(x, y):=\left(x^{\prime} \sqcap(x \sqcup y)\right) \sqcup\left(y^{\prime} \sqcap(x \sqcup y)\right) .
$$

Then

$$
t(x, x) \approx\left(x^{\prime} \sqcap(x \sqcup x)\right) \sqcup\left(x^{\prime} \sqcap(x \sqcup x)\right) \approx\left(x^{\prime} \sqcap x\right) \sqcup\left(x^{\prime} \sqcap x\right) \approx 0 \sqcup 0 \approx 0,
$$


and if $t(x, y)=0$ then $x^{\prime} \sqcap(x \sqcup y)=y^{\prime} \sqcap(x \sqcup y)=0$ and hence

$$
x=x \sqcup 0=x \sqcup\left(x^{\prime} \sqcap(x \sqcup y)\right)=x \sqcup y=y \sqcup x=y \sqcup\left(y^{\prime} \sqcap(y \sqcup x)\right)=y \sqcup 0=y .
$$

If we put

$$
\begin{aligned}
& t_{1}(x, y, z):=t(x, y) \sqcup z, \\
& t_{2}(x, y, z):=(t(x, y))^{\prime} \sqcap z
\end{aligned}
$$

then

$$
\begin{aligned}
& t_{1}(x, x, z) \approx t(x, x) \sqcup z \approx 0 \sqcup z \approx z, \\
& t_{2}(x, x, z) \approx(t(x, x))^{\prime} \sqcap z \approx 0^{\prime} \sqcap z \approx 1 \sqcap z \approx z,
\end{aligned}
$$

and if $t_{1}(x, y, z)=t_{2}(x, y, z)=z$ then $t(x, y) \leq z \leq(t(x, y))^{\prime}$ and hence $t(x, y)=$ $t(x, y) \sqcap(t(x, y))^{\prime}=0$ whence $x=y$. (Observe that in $\mathcal{L} c$ we have $0^{\prime} \approx 0 \sqcup 0^{\prime} \approx 1$.)

Finally, we are going to show the independence of identities (1) - (4).

Theorem 7. Within the variety of bounded $\lambda$-lattices with a unary operation the following hold:

(i) Identities (1) - (4) are independent,

(ii) identities (1) - (4) do not imply identity (5).

\section{Proof.}

(i) The $\lambda$-lattice $(\{0,1\}, \sqcup, \sqcap, 0,1)$ with

$$
\begin{array}{l|ll}
x & 0 & 1 \\
\hline x^{\prime} & 0 & 0
\end{array}
$$

satisfies (2), (3) and (4), but not (1) since

$$
(0 \sqcup 0) \sqcup\left(0^{\prime} \sqcup 0\right)=0 \sqcup(0 \sqcup 0)=0 \neq 1 .
$$

The $\lambda$-lattice $(\{0,1\}, \sqcup, \sqcap, 0,1)$ with

$$
\begin{array}{l|ll}
x & 0 & 1 \\
\hline x^{\prime} & 1 & 1
\end{array}
$$

satisfies (1), (3) and (4), but not (2) since

$$
(1 \sqcap 1) \sqcap\left(1^{\prime} \sqcap 1\right)=1 \sqcap(1 \sqcap 1)=1 \neq 0 .
$$


The $\lambda$-lattice shown in Fig. 6

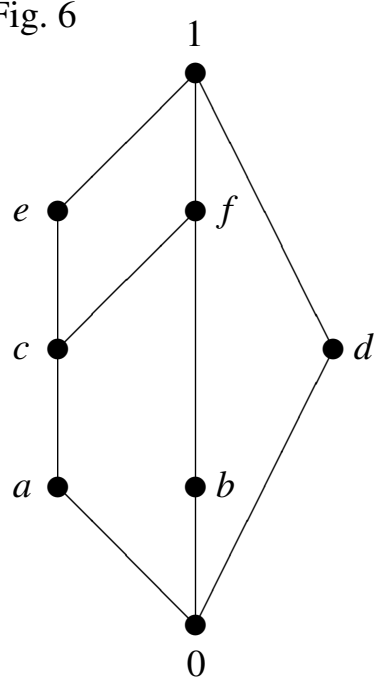

Fig. 6

with $a \sqcup b=1, b \sqcup c=f, e \sqcap f=c$ and

$$
\begin{array}{l|llllllll}
x & 0 & a & b & c & d & e & f & 1 \\
\hline x^{\prime} & 1 & d & e & d & a & b & d & 0
\end{array}
$$

satisfies (1), (2) and (4), but not (3) since $\left(\left(\left(a \sqcap b^{\prime}\right) \sqcup f\right) \sqcup(b \sqcup f)\right) \sqcap\left(\left(a \sqcap b^{\prime}\right) \sqcup b\right)=(((a \sqcap e) \sqcup f) \sqcup f) \sqcap((a \sqcap e) \sqcup b)=$ $=((a \sqcup f) \sqcup f) \sqcap(a \sqcup b)=(f \sqcup f) \sqcap 1=$ $=f \neq 1=a \sqcup b=(a \sqcap e) \sqcup b=$ $=\left(a \sqcap b^{\prime}\right) \sqcup b$.

The $\lambda$-lattice shown in Fig. 7

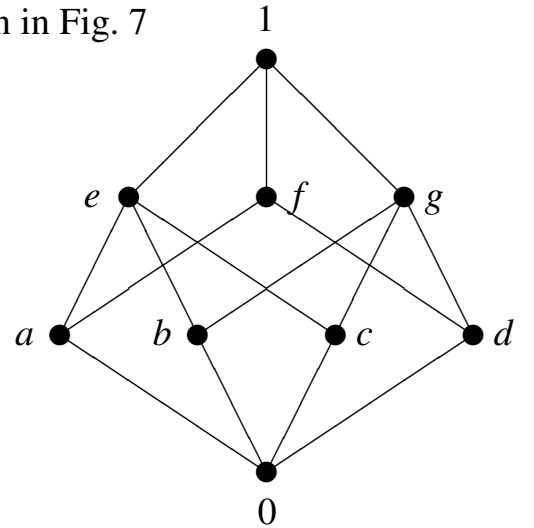

Fig. 7 
with $a \sqcup b=e, a \sqcup c=e, a \sqcup d=f, b \sqcup c=1, b \sqcup d=g, c \sqcup d=g, e \sqcap f=a$, $e \sqcap g=c, f \sqcap g=d$ and

$$
\begin{array}{l|lllllllll}
x & 0 & a & b & c & d & e & f & g & 1 \\
\hline x^{\prime} & 1 & g & f & f & e & d & b & a & 0
\end{array}
$$

satisfies (1), (2) and (3), but not (4) since

$$
\begin{aligned}
\left(\left((d \sqcap g)^{\prime} \sqcap b\right) \sqcap(g \sqcap b)\right) \sqcup\left((d \sqcap g)^{\prime} \sqcap g\right) & =\left(\left(d^{\prime} \sqcap b\right) \sqcap b\right) \sqcup\left(d^{\prime} \sqcap g\right)= \\
& =((e \sqcap b) \sqcap b) \sqcup(e \sqcap g)=(b \sqcap b) \sqcup c= \\
& =b \sqcup c=1 \neq c=e \sqcap g=d^{\prime} \sqcap g= \\
& =(d \sqcap g)^{\prime} \sqcap g .
\end{aligned}
$$

(ii) The $\lambda$-lattice shown in Fig. 8

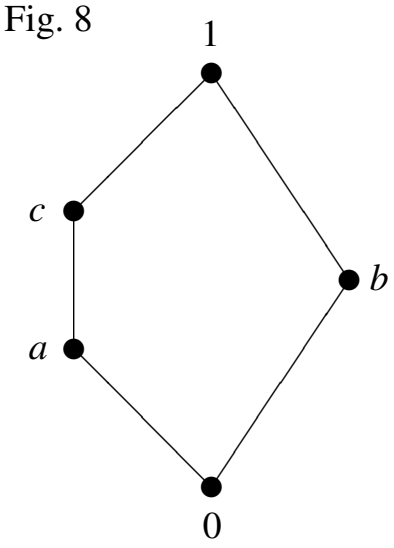

Fig. 8

with

$$
\begin{array}{l|lllll}
x & 0 & a & b & c & 1 \\
\hline x^{\prime} & 1 & b & a & b & 0
\end{array}
$$

satisfies (1) - (4), but not (5) since

$$
a \sqcup\left(a^{\prime} \sqcap(a \sqcup c)\right)=a \sqcup(b \sqcap c)=a \sqcup 0=a \neq c=a \sqcup c .
$$

It should be mentioned that the $\lambda$-lattices in Fig. 6 (with $a \vee b=f$ ) and in Fig. 8 are in fact lattices.

\section{REFERENCES}

[1] L. Beran, "Orthomodular lattices. Algebraic approach." Mathematics and Its Applications (East European Series), 18. Dordrecht - Boston - Lancaster: D. Reidel Publishing Company, a member of the Kluwer Academic Publishers Group; Prague: Academia, Publishing House of the Czechoslovak Academy of Sciences. XIX, 394 p., 1985, doi: 10.1007/978-94-009-5215-7.

[2] G. Birkhoff and J. von Neumann, "The logic of quantum mechanics." Ann. Math. (2), vol. 37, pp. 823-843, 1936. 
[3] S. Bonzio and I. Chajda, "A note on orthomodular lattices." Int. J. Theor. Phys., vol. 56, no. 12, pp. 3740-3743, 2017, doi: 10.1007/s10773-016-3258-6.

[4] G. Bruns and J. Harding, "Algebraic aspects of orthomodular lattices." in Current research in operational quantum logic. Algebras, categories, languages. Workshop, Free Univ. of Brussels, Belgium, June of 1998 and May of 1999. Dordrecht: Kluwer Academic Publishers, 2000, pp. $37-65$.

[5] I. Chajda, G. Eigenthaler, and H. Länger, "Congruence classes in universal algebra." Lemgo: Heldermann Verlag, 2012, pp. $\mathrm{x}+217$.

[6] I. Chajda and H. Länger, "Directoids. An algebraic approach to ordered sets." Lemgo: Heldermann Verlag, 2011, p. 176.

[7] I. Chajda and H. Länger, "Weakly orthomodular and dually weakly orthomodular lattices." Order, vol. 35, no. 3, pp. 541-555, 2018, doi: 10.1007/s11083-017-9448-x.

[8] I. Chajda and H. Länger, "Weakly orthomodular and dually weakly orthomodular posets." Asian-Eur. J. Math., vol. 11, no. 2, pp. $1850093-1-1850093-18,2018$, id/No 1850093, doi: 10.1142/S1793557118500936.

[9] K. Husimi, "Studies on the foundation of quantum mechanics. I." Proc. Phys.-Math. Soc. Japan, III. Ser., vol. 19, pp. 766-789, 1937.

[10] V. Snášel, “入-lattices.” Math. Bohem., vol. 122, no. 3, pp. 267-272, 1997.

Authors' addresses

Ivan Chajda

Palacký University Olomouc, Faculty of Science, Department of Algebra and Geometry, 17. listopadu 12, 77146 Olomouc, Czech Republic

E-mail address: ivan.chajda@upol.cz

\section{Miroslav Kolařík}

Palacký University Olomouc, Faculty of Science, Department of Computer Science, 17. listopadu 12, 77146 Olomouc, Czech Republic

E-mail address: miroslav.kolarik@upol.cz

\section{Helmut Länger}

(Corresponding author) TU Wien, Faculty of Mathematics and Geoinformation, Institute of Discrete Mathematics and Geometry, Wiedner Hauptstraße 8-10, 1040 Vienna, Austria, and Palacký University Olomouc, Faculty of Science, Department of Algebra and Geometry, 17. listopadu 12, 77146 Olomouc, Czech Republic

E-mail address: helmut. laenger@tuwien.ac.at 